Research Journal of Applied Sciences 13 (9): 513-521, 2018

ISSN: $1815-932 \mathrm{X}$

(C) Medwell Journals, 2018

\title{
High Resolution Spectroscopy in Sub-Meter Sized Optical Telescopes Using the Lhires III Spectrograph
}

\author{
Ivan Dario Arellano, Jairo Alberto Aguirre, Angelica Maria Guapacha and Edwin Andres Quintero \\ Department of Physics, Astronomical Observatory, Technological University of Pereira,
}

Pereira, Colombia

\begin{abstract}
High resolution optical spectroscopy is an instrumental technique of great applications in modern astronomy for its ability to obtain physical information such as: monitoring the activity of Nova stars, monitoring be stars pulsations, determining radial velocities eclipsing multiple stellar systems among others. However, given the instrumental complexity involved in high resolution spectroscopy, this technique is generally limited to large observatories with $1 \mathrm{~m}$ or more class telescopes which makes it difficult for amateurs and smaller observatories to participate in observation campaigns of this type of phenomena that take place worldwide. In this study we describe a methodology to acquire, process and obtain physical information from acquired spectra without requesting a very expensive instrumentation. The proposed methodology is tested with the equipment available at the Astronomical Observatory of the Technological University of Pereira, Colombia. Reduced and calibrated spectral profiles are obtained in wavelength and normalized flux, from the Sun, Jupiter and the eclipsing binary star systems $\beta$ Aurigae and $\delta$ Capricorni. The results obtained show that the proposed methodology allows users to obtain reduced and calibrated high-resolution spectra, captured with low cost instrumentation, thereby opening the possibility for small observatories and Amateur astronomers to contribute to the study of different astronomical phenomena of relevant interest.
\end{abstract}

Key words: High resolution spectroscopy, Lhires III, small telescopes, IRAF, low cost, small, spector

\section{INTRODUCTION}

High resolution optical astronomical spectroscopy is an instrumental technique widely used by large observatories around the world to determine physical variables of celestial bodies. However, the number of celestial bodies of interest exceed the optical capacity installed in the world, meaning that many of these are not monitored regularly (Garcia, 2010). To solve the lack of instrumentation for making astronomical observations it is possible to use sub-meter sized telescopes to capture stellar spectra. However, most of the spectrographs available for these telescopes outweigh the economic availability of many medium-sized observatories (Hopkins, 2014). Among commercial spectrographs, price vary: the eShel 9886 USD, the Baches 8179 Euros (Baches, 2017), the SBIG ST-I 795 USD (Sbig, 2017), the Lisa 3954 USD, the Starlight Xpress 3395 USD and the Lhires III 3954 USD. From the above mentioned only the eShel $(\mathrm{R}=10000)$, the Baches $(\mathrm{R}=18000)$ and the Lhires III $(\mathrm{R}=18000)$ spectrographs are high resolution which makes the Lhires III the most economical and best option in the market.
At the beginning of the 21 st century, a new opportunity for spectroscopy was opened when the French company Shelyak instruments designed the high-resolution Lhires III spectrograph. Up to that time, spectrographs for the amateur astronomers were of very low resolution and could not be adapted to sub-meter sized telescopes (between 8 and 20 inches). Therefore, non-professional observatories lacked instruments to make important contributions to observational astronomy and Shelyak Instruments provided the opportunity to change this (Thizy and Cochard, 2010). The high-resolution spectrograph Lhires III opens the possibility to do research for astronomical observatories with sub-meter sized telescopes. An example of this is the ARAS (Astronomical Ring for Access to Spectroscopy) project. The aim of this project is the generation of spectral databases using joint observations between professionals and amateurs, regulated by the BeSS spectra database (database of stars type be, Nova, hot massive stars) (Thizy et al., 2008; Thizy, 2008) or possible collaborations with organizations such as the AAVSO (Anonymous, 2017a-d; Gorodenski, 2012).

Corresponding Author: Ivan Dario Arellano, Department of Physics, Astronomical Observatory, Technological University of Pereira, Pereira, Colombia 
At Hard Labor Creek Observatory (HLCO) in Georgia, Jenkins (2011) studied the characteristics and the tuning of the Lhires III spectrograph in a 20 inch RCT (Ritchey-Chretien Telescope). The wavelength calibration was performed for three diffraction gratings: 600, 1200 and 2400 grooves $/ \mathrm{mm}$ considered low, medium and high resolution, respectively. The internal calibration Neon lamp of the equipment was used for the comparison spectra. These were processed in IRAF. Using the star HD 116658 ( $\alpha$ Virgo), the authors demonstrate the enormous potential of the Lhires III to do high-resolution spectroscopy with a sub-meter sized telescope.

At the Hopkins Phoenix Observatory (HPO) in Arizona, Hopkins (2012) performed spectroscopic observations of the star $\mathrm{HD} 31964$ ( $\varepsilon$ Aurigae) with two small telescopes, 8 and 12 inches and the high-resolution Lhires III spectrograph. By taking spectra of the star and measuring the Balmer $\left(\mathrm{H}_{\alpha}, \mathrm{H}_{\beta}, \mathrm{H}_{\gamma}, \mathrm{H}_{8}\right.$ and $\left.\mathrm{H}_{\varepsilon}\right)$ and sodium lines, they calculated the radial velocity of the system.

This demonstrates that the physical data that can be obtained with this instrumental arrangement (sub-meter sized telescope and Lhires III) are significant.

\section{MATERIALS AND METHODS}

The Astronomical Observatory of the Technological University of Pereira (AOTUP) is located in Colombia, at the coordinates $4^{\circ} 47^{\prime} 25.79^{\prime \prime} \mathrm{N} 75^{\circ} 4125.48^{\prime \prime} \mathrm{W}$ and an altitude of 1350 m.a.s.l. It has a telescope Meade LX200GPS 16", a SBIG STF-8300M camera and the Lhires III spectrograph. In order to take high resolution spectra with the instrumentation available at the AOTUP, a technique was implemented; the problem was divided in several phases and subphases, according to the used instrumentation and implemented solution respectively. The implementation of the methodology was carried out following the optical path of the light in the equipment for which in the present study it is addressed in this same order as seen in the block diagram of Fig. 1. In the design of a Lhires III spectrograph, the light enters through the telescope, part of which passes through the slit of nominal size $25 \mu \mathrm{m}$ and another part which is reflected to the guide camera. The beam passing through the slit is sent to the collimator lens by the $45^{\circ}$ main mirror. The collimated beam then passes to the diffraction grating and the scattered light passes back through the lens and is focused on the CCD detector of the main camera, according to Fig. 2.

Measuring the slit width: The resolution of the spectra is determined by the slit width. If it is bigger, the resolution decreases. If it is smaller, some flux is lost. Since, the slit fabrication is not ideal it should be adjusted as precisely as possible. This can be done by applying the rules of optics (Alonso and Finn, 1998). By means of the diffraction of Fraunhofer it is possible to calculate the slit width (Eq. 1):

$$
\alpha=\frac{\mathrm{D} \lambda}{\mathrm{x}}
$$

Where:

$\alpha=$ The width of the rectangular slit

$\lambda=$ The wavelength of the laser

$\mathrm{D}=$ The distance from the slit to the area of projection of the diffraction pattern

$\mathrm{x}=$ The distance from the center of the diffraction pattern to the first minimum For the slit width, we found $23.52 \pm 0.12 \mu \mathrm{m}$

Collimation of the optical elements of the spectrograph: Collimation of optical elements includes alignment of the diagonal mirror of the guiding camera, alignment of the spectrograph's main mirror and focus of the collimator lens to achieve a clear image in the main camera. Firstly, the light of the study body that passes through the telescope should be focused on the spectrograph slit. For this purpose, the Lhires III slit is made on a reflective surface that redirects the light to a diagonal mirror which projects the image to the guide camera. The camera is positioned in such a way that the slit is in the axis of the Right Ascension (RA) movement of the telescope. The Bigourdan method is implemented to obtain exposures greater than $10 \mathrm{~min}$ without drift due to tracking errors of the mount (Martinez, 1994). The errors that persist are corrected using a tracking software that corrects the drift of the field by synchronizing a reference star seen in the guide camera. In this, MaxIm DL 6 was used to perform those corrections.

\section{Determination of the equation of the diffraction grating:}

The order of the spectrum observed in the CCD camera is the function of the inclination in which the light of the object of study falls on the diffraction grating and of the angle with which it is reflected, according to the Eq. 2:

$$
\mathrm{m} \lambda=\mathrm{d}(\sin \alpha+\sin \beta) \mathrm{m}=0, \pm 1, \pm 2, \ldots
$$

Where:

$\alpha=$ The angle of incidence of the light

$\beta=$ The diffraction angle

$\lambda=$ The wavelength

$\mathrm{m}=$ The diffraction order

$\mathrm{d}=$ The spacing between the adjacent grooves on the surface of the grating 


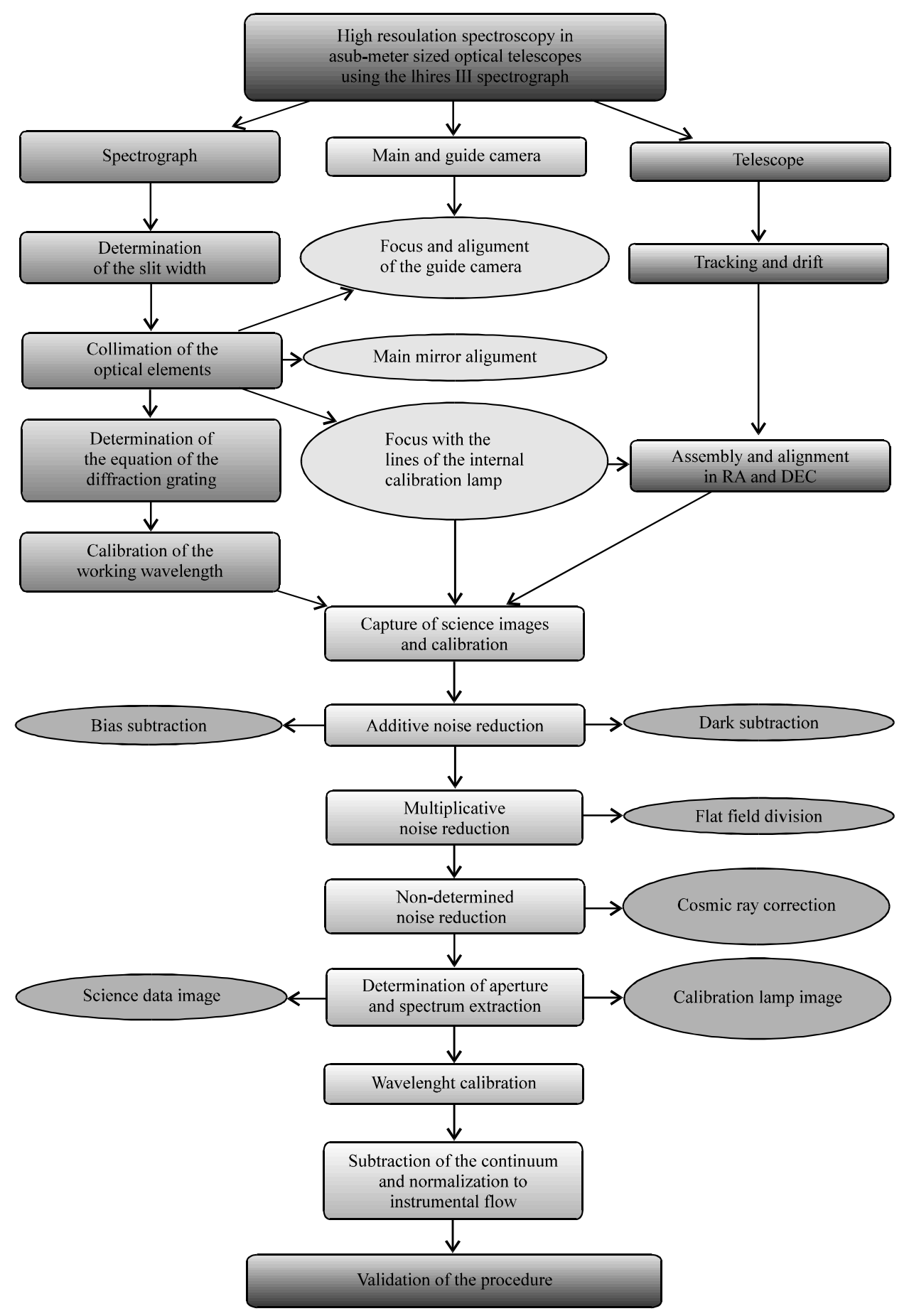

Fig. 1: General diagram of the proposed methodology

The Lhires III spectrograph has a Littrow configuration in which light travels back along the same direction of the incident light; therefore in Eq. $2, \alpha=\beta$ and the equation is reduced to Eq. 3 which is the function of the inclination of the grating itself. This inclination is controlled by a micrometric screw that must be calibrated. spectral lines of lamps such as mercury (Hg), Argon (Ar), Neon $(\mathrm{Ne})$ and Hydrogen $(\mathrm{H})$ were taken. The images were captured with the SBIG STF-8300M in such a way that each spectral line remains in the central part of the image. Figure 3 shows the calibration curve obtained. The black dots correspond to the values taken from the position of 


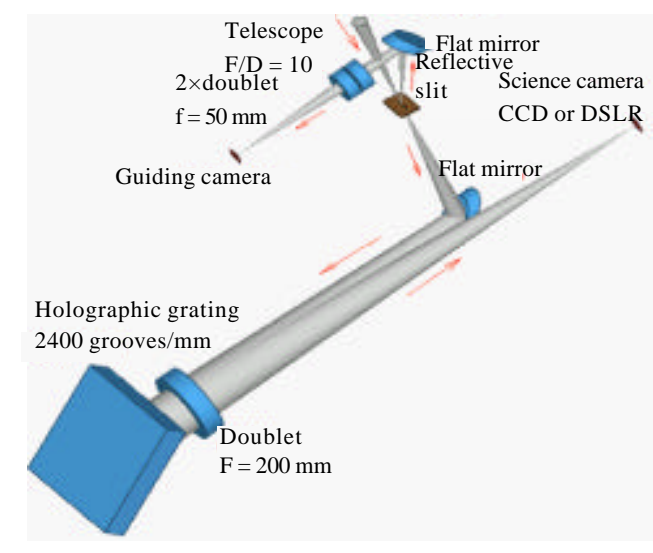

Fig. 2: Optical path of light in the Lhires III spectrograph

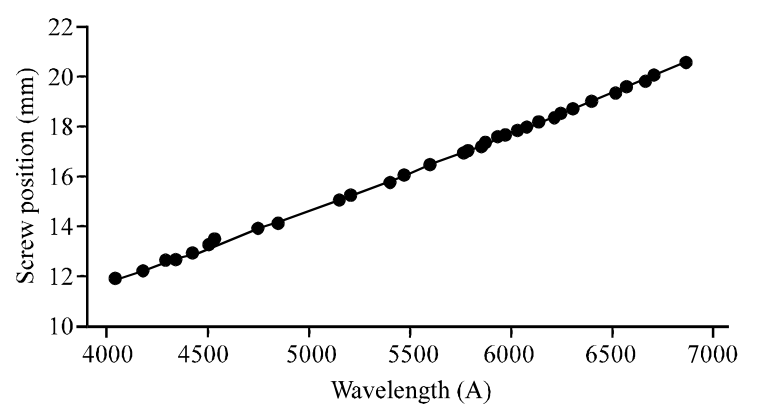

Fig. 3: Calibration curve of the Lhires III spectograph

the micrometric screw and the blue line corresponds to the estimation of the measurements. This was done implementing a MATLAB routine, finding a polynomial relation of order 2 (Eq. 4):

$$
p(x)=1.179 \times 10^{-7} x^{2}+1.754 \times 10^{-3} x+2.866
$$

The calculation of the uncertainty in the position of the micrometric screw given by Eq. 4 was made through covariance matrix. Since, the uncertainty value is variable for each position, a routine was implemented in MATLAB called posicion that shows the position in which the micrometric screw must be placed for a chosen wavelength with an uncertainty value. A software called Lambda for Lhires III was developed to perform the calculation of the observed wavelength from the position of the micrometric screw and vice versa. Although, in the literature there are different references in which the relationship between the micrometric screw and the wavelength is considered linear (Hopkins, 2014; Rosas et al., 2017) based on the observation of the behavior of this system it was established that this relationship is non-linear. The Software Lambda for Lhires
III considers this non-linearity and makes a reconstruction by the least squares method. In this way, the problem of non-linearity between the position of the screw and the expected wavelength was solved.

Calibration and acquisition of science images: The image of the object of study is captured with an exposure time according to the magnitude of the object. This is calculated with the Exposure Time Calculator for Lhires III (ETCL). This is an application developed by the French manufacturer Shelyak instruments as a script for excel in which the characteristics of the telescope, the slit of the spectrograph, the diffraction grating of the spectrograph, the camera and the magnitude of the object are entered. For the capture of the science, image, a sequence of images with a fixed exposure value of time, according to the ETCL calculation is initially taken. In practice it has been observed that the exposure time is approximated and should be increased by $10 \%$ to obtain viable images. This is due to the fact that ETCL calculates this exposure time only considering the instrumental setup and not bearing in mind atmospheric effects seeing, extinction coefficient and the sky background magnitude.

To properly correlate the position of the field of interest with the studied wavelength after completion of the previous acquisitions, exposures of the internal calibration lamp should be made (Fig. 4). These images should be taken with an adequate exposure time, so that, the main lines do not become saturated while ensuring other useful identification lines are visible. It is important to see between 2 and 3 lines in the field without saturation in order to proceed with the dispersion correction of the field of the object of study. To ensure accuracy it is recommendable to take these images at the beginning and the end of the observation day to observe any variation of the wavelength that may have occurred during the observation.

Reduction of additive, multiplicative and non-determined noises: The additive noise (Bias and Darks) of the science images were reduced automatically by the software that captures the images. In this experiment, MaximDL V. 6 was used. For the case of the flat field normalization, all the captured flat frames were usually taken before the beginning of the sequence of the science image. All science and flats images were processed using the software, Image Reduction and Analysis Facility (IRAF) V2.16. The flats were stacked in a single frame using the imcombine task, creating an image called "Master Flat". Then all the science images were divided by the Master Flat with the imarith task to eliminate the multiplicative 


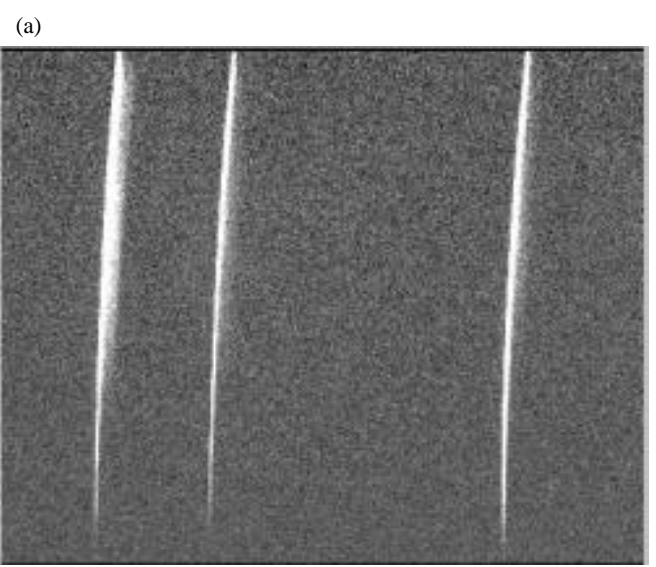

(b)

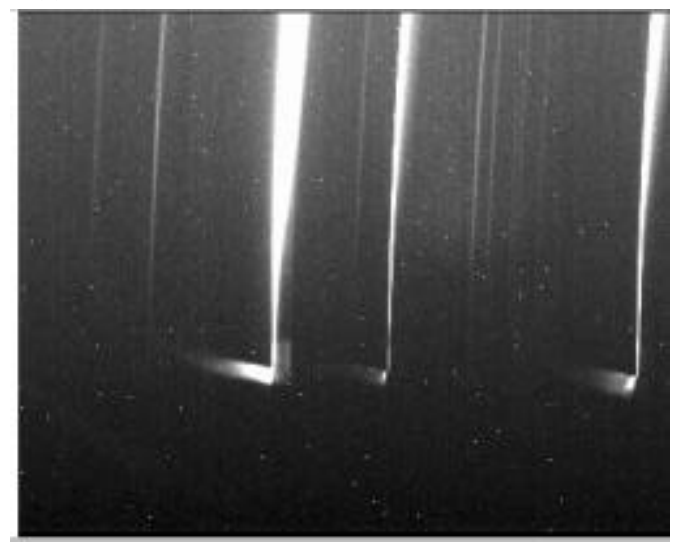

Fig. 4: Images of the neon lamp acquired with $2 \mathrm{sec}$ of exposure to process the images of; a) Jupiter in the region of 5770 and $5950 \AA$ and b) HD40183 in the region of 6480 and $6650 \AA$

noise. Even after processing the images, there are pixels with values outside the average. Some of these may be due to the presence of cosmic rays, so, from the individual images the noise present was eliminated with the cosmicrays task to obtain a science image as clean as possible. The task eliminates all the noise pixels that have not yet been corrected and with that, we obtain the final science image to extract the spectra.

Extraction of the spectrum: The science images filtered of all the present noises are reviewed individually and those with the best characteristics (SNR and alignment correction) are combined in a single final image with the imcombine task. This obtained image contains the 2D spectrum of the study object. Henceforth, we will call it the combined science image. Figure 5 and 6 show the 2D spectrum of the study objects of this study. To extract the spectral profile from the combined science image of the study objects, the apall task is applied. In this image, the region where the data are found is selected and identified then, the region of the image corresponding to the

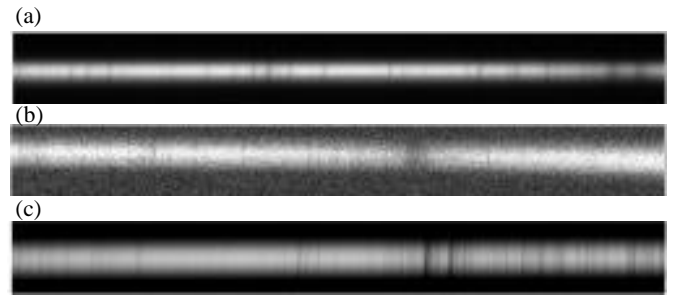

Fig. 5: 2D spectra profile of; a) $\beta$ Aurigae; b) $\delta$ Capriconi and c) Jupiter

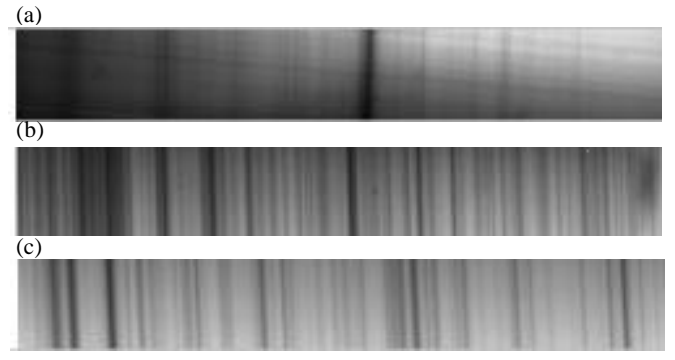

Fig. 6: 2D spectra profile of the sun; $\mathrm{H} \alpha, \mathrm{b}) \mathrm{H} \gamma$ and c) $\mathrm{Mg}$ lines

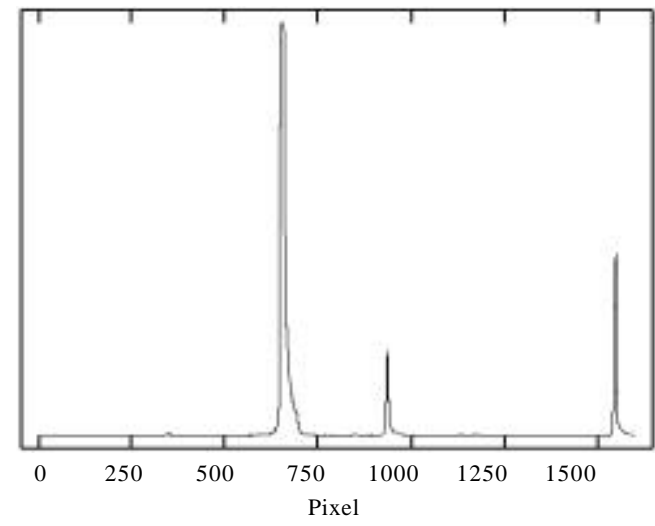

Fig. 7: Spectral profile of the neon lamp used for the calibration of the planet ajupiter; NOAO/IRAF V2.16 alfaorion@ALFARION Fri 01:37:39 02-Dec-2016 sepration step $=0$

background is identified and finally in order to obtain the best curve, one of the mathematical functions (spline, spline 3 , legendre, chebyshev) is applied to the dispersion of data available in IRAF. The aforementioned process allows astronomers to obtain the spectral profile (1D) of the object of study. The axis of this profile is in pixels vs counts, so to calibrate it it is necessary to extract the spectra of the calibrating lamps exactly on the same axis of dispersion of the combined science image. In order to do this, the calibration lamp images were stacked in a single image using the imcombine task. After this, the apall task was applied to extract the 1D spectrum of the calibration lamp (Fig. 7) and was proceeded to indicate the 
wavelength of each spectral line by means of the command identify. At this point, the observed wavelength scale is obtained and it is assigned to the $\mathrm{x}$ axis of the 1D spectrum of the science image by means of the dispcor task.

\section{RESULTS AND DISCUSSION}

The proposed methodology presented in Fig. 1 was tested by capturing, pre-processing and reducing the spectra of the Sun, the planet Jupiter and the eclipsing binary spectroscopy stars $\delta$ Capricorni and $\beta$ Aurigae.

Sun: Without a doubt, the Sun was the first celestial body studied from the spectroscopy. Today, the solar spectrum is widely used in the calibration and tuning of astronomical instrumentation and in the teaching of spectroscopy. Figure 8 and 9 show the solar spectrum calibrated in wavelength and normalized to instrumental flow. They were obtained by applying the methodology proposed in this article and are centered on the wavelength $\mathrm{H} \alpha(6563 \AA)$ and $\mathrm{H} \gamma(4341 \AA)$, respectively. Likewise, Fig. 10 shows the solar spectrum obtained between 5170 and $5350 \AA$. Note the spectral lines associated with $\mathrm{Mg}$ and $\mathrm{Fe}$. The high resolution solar spectroscopy is currently used to study the composition of the solar corona during total eclipses (Mumpuni et al., 2016), so, the present methodology opens the possibility for amateur observatories to contribute to knowledge about this solar phenomenon.

Jupiter: From the pre-processing and reduction of the image shown in Fig. 5c, we were able to obtain Fig. 11 which shows the spectrum for the planet Jupiter between 5770 and $5950 \AA$. Note the well-defined sodium lines D1 $(5890 \AA)$ and D2 $(5896 \AA)$, one of the main characteristics of the spectrum of this planet that is associated with the absorption made by this element present in the atmosphere of Jupiter (Firsoff, 1968; Trypsteen and Walker, 2017). The final spectrum is instrumental flow normalized with subtraction of the continuum and calibrated in wavelength. In long time exposure spectra, the spectral bands of the planets show a slight inclination (only observable in high resolution spectroscopy) associated with the Doppler effect caused by the rotation of the planets (Acker and Jaschek, 1981). Therefore when considering this inclination from the observation of the spectrum it is possible to calculate the rotation period which is constituted as an application of high resolution spectroscopy, widely used for educational purposes. Thus, the methodology proposed in this article can be used for the creation of laboratory practices that allow undergraduate students in physics and astronomy to

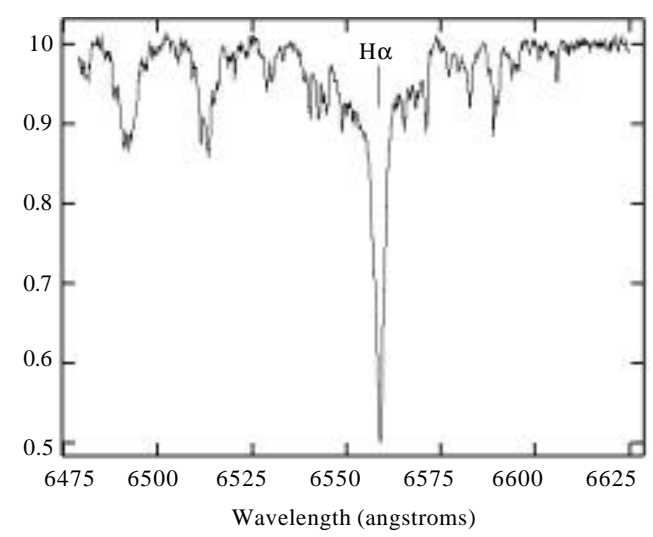

Fig. 8: Spectral profile of the sun in the region between 6475-6625 Å; NOAO/IRAF V2. 16 alfaorion@, AlFAORION Thu 11:41:09 [sol-hafa-calnorm.fits]: 20. ap: 1 beam: 1

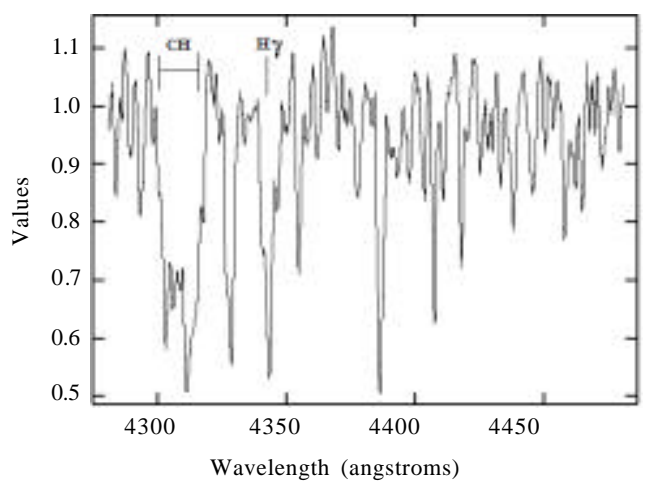

Fig. 9: 1D spectrum of the sun centred on the wavelength Hy; NOAO/IRAF V2.16 alfaorion@ ALFAORION Thu 18:23:13 [sol-hgamma- calnorm. fits]: 10. ap:1 beam:1

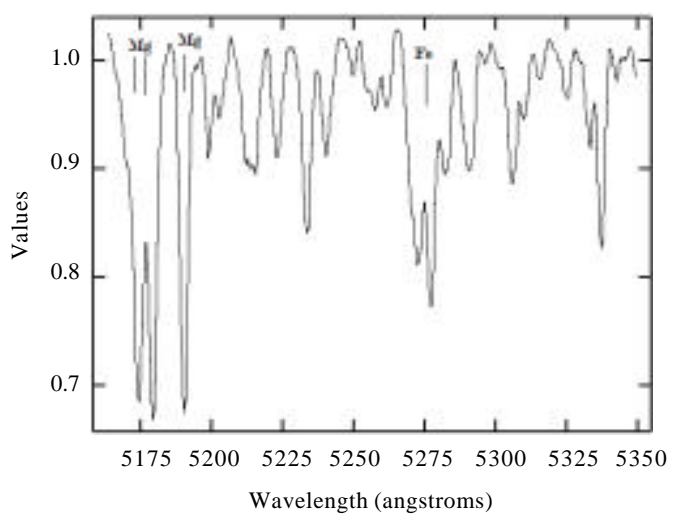

Fig. 10: Spectral profile of the sun showing the $\mathrm{Mg}$ and fe lines; NOAO/IRAF V2.16 alfaorion@ALFARION Mon 20:44:47 [sol-hierro-calnom.fits]: 10. ap:1 beam: 1 


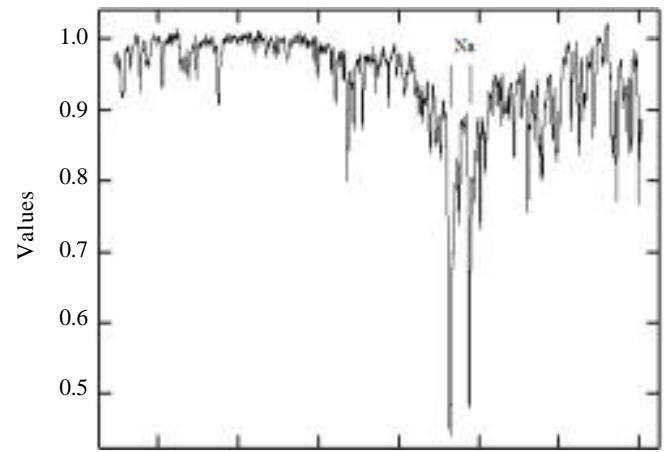

Wavelength (angstroms)

Fig. 11: Jupiter spectral profile in the region 5770-5950 NOAO/IRAF V2.16 alfaorion@ALFARION Tue 15:30:28 11-Oct-2016 [Jupiter-cal-lo.fi.FIT]: 240. ap: 1 beam: 1

interact with concepts and procedures of high resolution spectroscopy and its application in the solution of common problems in astronomy such as the calculation of rotation periods.

Beta Aurigae: $\beta$ Aurigae (HD 40183) is an eclipsing binary spectroscopy star with an orbital period of 3.96 days (Southworth et al., 2007). Fig. 12 shows the 1D spectrum calibrated in wavelength and normalized in instrumental flux obtained for this binary star, centered at the wavelength of $\mathrm{H} \alpha(6563 \AA)$. The observed doublet lines correspond to the absorption in the stellar atmosphere associated with the 3-2 transition of hydrogen in each of the stars that make up this binary system. Meanwhile, Fig. 13 shows the spectrum of the lamp used to calibrate the spectrum of this binary star.

Delta Capricorni: $\delta$ Capricorni (HD 207098) is also an eclipsing binary spectroscopic star with an orbital period of 1.022 days (Wonnacott et al., 1992). Figure 14 shows the 1D spectrum calibrated in wavelength and normalized in instrumental flux, centered at the $\mathrm{H} \alpha$ line. In this case, the absorption lines of the two stars coincide since, the observation was made in an instant of the orbital period in which one of the stars is at the closest point to the Earth while the other is at the farthest point (eclipse position), generating the superposition of the spectra. From the displacement by the Doppler effect of each of the lines on the wavelength axis and throughout the orbital period it is possible to construct the radial velocity curve of both $\beta$ Aurigae and $\delta$ Capricorni systems. By means of this curve it is possible to determine the parameters that describe the orbital dynamics of the binary set (Galeotti and Guerrero, 1968; Smith, 1948;

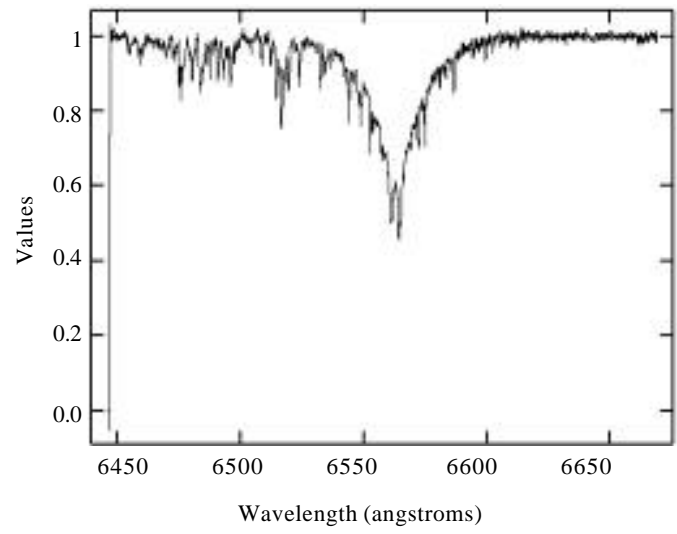

Fig. 12: Spectral profile of the Beta Aurigae atar in the region of 6450-6650 $\AA$; NOAO/IRAF V2.16 alfaorion@ALFARION Fri 15:45:52 05-May-2017 [20170205hd40183-0031.wl.n.fits]: HD40813240. ap: 1 beam:1

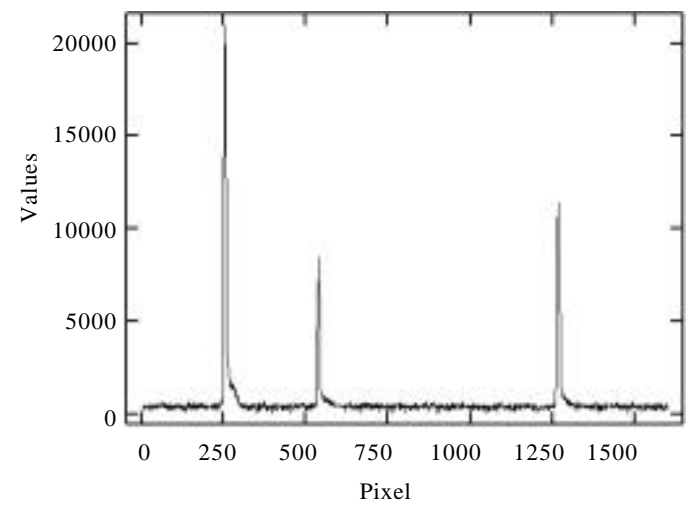

Fig.13: Spectral profile of the neon lamp used for the calibration of beta Aurigae star; NOAO/IRAF V2.16 alfaorion@ALFARION Fri 18:52:50 02-Dec2016 [arco-0001 fits]: 2 ap:1 beam:1

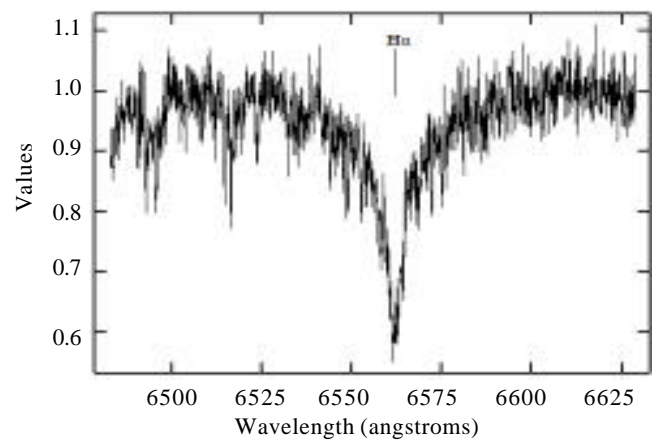

Fig. 14:1D spectrum of $\delta$ Capriconi star in the region of 6475-6625 A; NOAO/IRAF V2.16 alfaorion@ ALFARION Thu 16:27:57 20-Oct-2016 [sepcdelcap-calibradol.fits]: 1200. ap:1 beam: 1 
Slipher, 1906). As mentioned in the introduction, this procedure is one of the applications of high resolution spectroscopy. Therefore, the methodology described in this study can be used, so that, observatories with sub-meter sized telescopes get involved in the study of eclipsing binary systems.

It has been demonstrated that it is possible with rigor in the process of instrumental and processing calibration such as that proposed in the methodology developed in this work, to acquire, process and obtain physical data from acquired spectra with sub-meter sized telescopes such as the Meade LX200 GPS telescope of $40.64 \mathrm{~cm}$ and the low-cost, high-resolution Lhires III spectrograph.

\section{CONCLUSION}

High resolution spectroscopy involves both instrumental and image processing aspects which make the implementation of this technique a complex task, usually exclusive to large observatories. For instance in the instrumental field, the correct collimation of the optical elements, the estimation of the wavelength in which the observation is done and the precise focus, alignment and monitoring of the object of study among others are crucial. As for the treatment of the images obtained, an appropriate reduction of the noise present in the images as well as the correct calibration and normalization of the spectral profiles are essential to obtain scientific data. The methodology described in this study includes all these aspects in an articulated manner in such a way that it makes it possible to obtain high-resolution spectra of sufficient quality to extract scientific information about different astronomical phenomena of relevant interest. We come to the conclusion that it is possible to do high resolution spectroscopy and reach valuable results using a low-cost instrumentation such as the Lhires III spectrograph and a sub-meter sized telescope. This opens the possibility for small observatories and amateur astronomers to have access to high resolution spectroscopy and in this way, participate in worldwide networks of observations of super novae and Be stars among others while making contributions to modern astronomy.

\section{REFERENCES}

Acker, A. and C. Jaschek, 1981. Astronomy: Methods and Calculations Corrected Exercises Paperback. Masson, Paris, France, Pages: 284.

Alonso, M. and E.J. Finn, 1998. [Physics: Fields and Waves: Volume 2 of Physics]. Pearson, London, UK.,.
Anontmous, 2017a. Baches Echelle-spectrograph. Baader Planetarium GmbH, Mammendorf, Germany. http://www.baader-planetarium.com/en/bachesechelle-spectrograph.html

Anonymous, 2017b. Can very small telescopespectrographs do useful work for AAVSO? American Association of Variable Star Observers, Cambridge, Massachusetts, USA. https://www. aavso.org/can-very-small-telescope-spectrographsdo-useful-work-aavso

Anonymous, 2017c. Starlight xpress reflective grating spectrograph with lodestar guide camera. Visa, Foster City, California, USA. https://optcorp. com/products/starlight-xpress-spectrograph-withlodestar-guide-camera

Anonymous, 2017d. [Welcome to Lambda for lhires III]. Technological University of Pereira, Pereira, Colombia. (In Spanish) https:// observatorioastronomico.utp.edu.co/noticias/lamb da-for-lhires.html

Firsoff, V.A., 1968. Exploring the Planets. AS Barnes, South Brunswick, New Jersey, USA.,

Galeotti, P. and G. Guerrero, 1968. Elementi spettroscopici della binaria Beta Aurigae. Memorie Della Soc. Astron. Ital., 39: 269-274.

Garcia, V.M.A., 2010. [Automatic determination of stellar parameters from high resolution spectra of cold stars]. Master Thesis, Complutense University of Madrid, Madrid, Spain. (In Spanish)

Gorodenski, S.A., 2012. High resolution spectroscopy for the amateur: Experiences with the LHIRES III spectrograph. Proceedings of the 31st International Annual Symposium on Society for Astronomical Sciences Vol. 31, May 22-24, 2012, Society for Astronomical Sciences, Rancho Cucamonga, California, USA., pp: 105-112.

Hopkins, J.L., 2012. Small telescope spectroscopy of Epsilon Aurigae. Proceedings of the 31st Annual International Symposium on Society for Astronomical Sciences Vol. 31, May 22-24, 2012, Society for Astronomical Sciences, Rancho Cucamonga, California, USA., pp: 89-96.

Hopkins, J.L., 2014. Lhires III Spectroscopy. In: Using Commercial Amateur Astronomical Spectrographs, Hopkins, J.L. (Ed.). Springer, Berlin, Germany, ISBN:978-3-319-01441-8, pp: 151-169.

Jenkins, B.G., 2011. A study of the lhires III spectrograph on the hard labor creek observatory 20 inch telescope. Master Thesis, Georgia State University, Atlanta, Georgia. 
Martinez, P., 1994. The Observer's Guide to Astronomy. Cambridge University Press, Cambridge, UK., Pages: 1149.

Mumpuni, E.S., M.Z. Nurzaman and N. Suryana, 2016. Preliminary results of the solar corona spectroscopic observation of 9th March 2016 total Solar Eclipse. J. Phys. Conf. Ser., 771: 1-4.

Rosas, F., J. Ordonez, W. Suarez and A. Quijano, 2017. Measurement of the Radial Velocity of Vega and SAO 104807 by High Resolution Spectrometry. In: Revista Mexicana de Astronomia y Astrofisica, Garzon M .A.H. and V.D. Santiago (Eds.). National Autonomous University of Mexico, Mexico City, Mexico, pp: 143-143.

Slipher, V.M., 1906. Variable radial velocity of delta Capricorni. Astrophys. J., Vol. 24, 10.1086/141403.

Smith, B., 1948. A spectroscopic study of beta Aurigae. Astrophys. J., 108: 504-509.

Southworth, J., H. Bruntt and D.L. Buzasi, 2007. Eclipsing binaries observed with the WIRE Satellite-II. â Aurigae and Non-linear limb darkening in light curves. Astron. Astrophys., 467: 1215-1226.
Thizy, O. and F. Cochard, 2010. Spectrographs for small telescopes International Astronomical Union. Proc. Intl. Astron. Union, 6: 282-283.

Thizy, O., 2008. Classical be stars high resolution spectroscopy. Proceedings of the 27th Annual Symposium on Society for Astronomical Sciences Vol. 27, May 20-22, 2008, Society for Astronomical Sciences, Rancho Cucamonga, California, USA., pp: $1-49$.

Thizy, O., C. Buil, F. Cochard and C. Neiner, 2008. Organizing a Professional-amateur collaboration with a Lhires III spectrograph. Planet. Space Sci., 56: 1878-1881.

Trypsteen, M.F.M. and R. Walker, 2017. Spectroscopy for Amateur Astronomers: Recording, Processing, Analysis and Interpretation. Cambridge University Press, Cambridge, UK., ISBN:9781 107166189, Pages: 162.

Wonnacott, D., B.J. Kellett, L. Matthews, C.A. Clayton and G.E. Bromage et al., 1992. ä Cap-the secondary revealed. Monthly Not. Royal Astron. Soc., 259: 251-258. 